\title{
Optimization of the Noise Transfer Function of Extended-Frequency-Band-Decomposition sigma-delta A/D converters
}

\author{
Ali Beydoun, Philippe Benabes, Jacques Oksman
}

\begin{abstract}
Frequency-Band-Decomposition (FBD) is a good candidate to increase the bandwidths of ADC converters based on sigma-delta modulators. Each modulator processes a part of the input signal band and is followed by a digital filter. In the case of large mismatches in the analog modulators, a new solution, called Extended Frequency-Band-Decomposition (EFBD) can be used. This solution allows for, for example, a four percent error in the central frequencies without significant degradation in the performance when the digital processing part is appeared to the analog modulators. A calibration of the digital part is thus required to reach these theoretical performance. This paper will focus on a self-calibration algorithm for an EFBD. The algorithm helps minimize the quantization noise of the EFBD.
\end{abstract}

\section{INTRODUCTION}

The Frequency-Band-Decomposition (FBD) [1], [2], [3] is a natural way to widen the bandwidth of sigma-delta converters [4], using parallel bandpass modulators, where each modulator processes a part of the input signal band [5]. The main issue of such a solution is its high sensitivity to the central frequencies of the bandpass modulators. Extended FrequencyBand-Decomposition (EFBD) [5] makes it possible to convert a band-limited signal, using $N+2$ parallel continuous-time bandpass modulators. This solution is able to adapt to the ana$\log$ mismatches of the modulators caused by variations in the manufacturing processes, in order to minimize the quantization noise of the system and to allow for the reconstruction of the input signal by a transfer function which is as close as possible to a simple delay (minimum in-band ripple and linear phase).

As in many conversion systems, a calibration of the digital part of the EFBD becomes unavoidable. In the case of TimeInterleaved converters, gain and offset errors induce unwanted tones that can be minimized by an adequate calibration [6] [7], [8]. Such a calibration is performed by applying constant signals to each modulator and deducing the gain and offset from the filter outputs.

One of the advantages of the FBD solutions is that linear mismatches do not produce spurious tones. They only result in an in-band ripple and in an increase of the quantization noise. Using continuous-time modulators helps increase the sampling frequency, and induces natural filtering in the work band of each modulator. The calibration proposed in [8] for FBD can only deal with offset and gain, which is not sufficient for EFBD solutions. An algorithm that can calibrate the digital part of an EFBD without requiring heavy computing resources is proposed in this paper.
The second section of this paper gives the theoretical elements of the calibration method. The third section presents the calibration algorithm : the output mean power is minimized by modifying the bandwidths of the filters, the input being simply grounded. Finally, the fourth section concludes with the complexity and the duration of the whole algorithm.

\section{Optimization OF EFBD Noise Transfer Function}

An EFBD is composed of $N+2$ parallel sigma-delta modulators (Fig. 1 - part A), where $N$ is the number of modulators required to process the input signal band $\left[f_{1} \ldots f_{2}\right]$. Two extra modulators are used in the case of large analog mismatches so that the useful band $f_{1} \ldots f_{2}$ remains within the work band of the EFBD. The outputs of all channels are merged by a digital system (part B) to reconstruct the input signal.

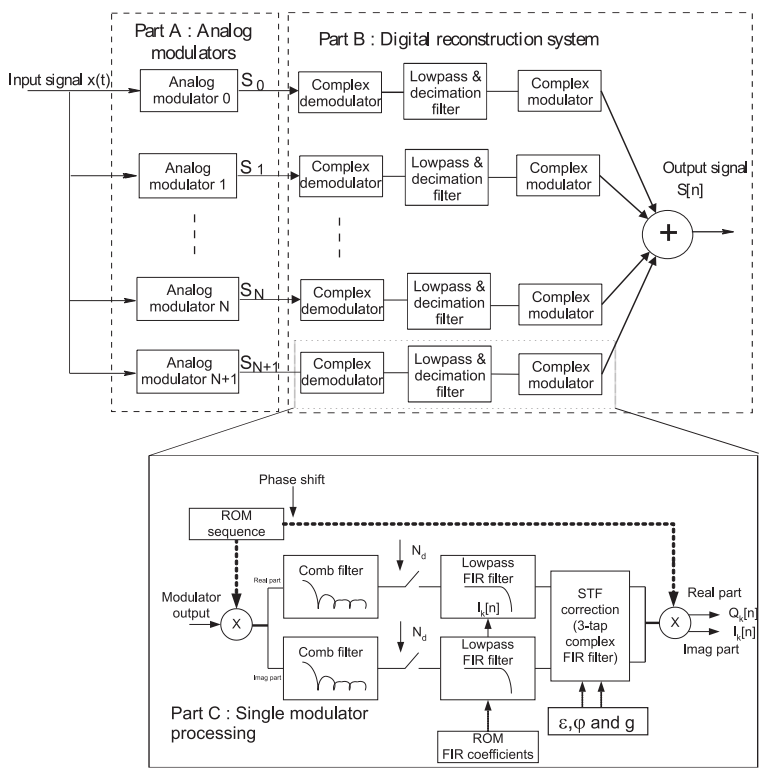

Fig. 1. Extended Frequency Band Decomposition architecture

The digital processing associated with each modulator is composed of a demodulation that brings the signal to baseband (demodulation frequencies are denoted $f_{C}^{k}$ ), of a comb filter that performs a decimation, of a FIR filter $H_{k}(z)$ that removes the out-of-band noise, of a signal transfer function correction $C_{1}(z)$ and of a modulation. The complete digital processing for one modulator is summarized in Fig. 1 - part C. The frequencies used for demodulation and modulation are 
expressed as rational numbers so that the sequences are finite and can be stored in a ROM [5].

The NTF optimization of the system consists in using, for each frequency band, the modulator that has the best signalto-noise ratio. As the signal transfer function is usually quite flat in the work band of the modulator, the modulator which is to be used for each frequency is the one whose noise power density is the lowest at this frequency.

Fig. 2 gives as an example, the noise power densities of each modulator $\left(N T F^{k}\right)$, where all resonator frequencies differ from their ideal values by a constant value plus random mismatches (modelling process non-idealities). The $\mathrm{Q}$ factors of the filters are equal to 50 . The boundaries between the band of each modulator are represented by vertical lines. In this example $\left(f_{1}=0.2, f_{2}=0.3\right)$, the $10^{\text {th }}$ modulator is not used as its band is completely outside the signal band.

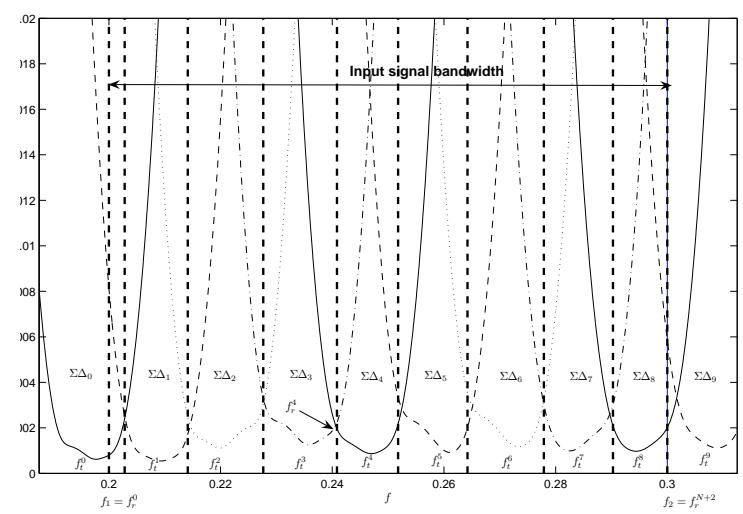

Fig. 2. Boundaries with non-ideal modulators

The global system quantization noise power is the sum of contributions of each modulator: $P_{N T F_{t}}=\sum_{k=0}^{N+1} P_{N T F^{k}}$.

$P_{N T F^{k}}=\int_{f=-\frac{1}{2}}^{\frac{1}{2}}\left|H^{k}\left(e^{2 j \pi N_{d}\left(f-f_{C}^{k}\right.}\right)\right|^{2}\left|N T F^{k}\left(e^{2 j \pi N_{d} f}\right)\right|^{2} \Gamma_{k}(f) d f$

$N_{d}$ is the decimation ratio, $\Gamma_{k}(f)$ is the quantization noise power spectral density and can be assumed constant [10]. $H_{k}(f)$ is the $k^{\text {th }}$ FIR filter transfer function. It is assumed that the decimation comb filter removes sufficiently the folded noise produced by aliasing.

Fig. 3 gives as an example the magnitude of the product $\left|H^{k}\left(e^{2 j \pi N_{d}\left(f-f_{C}^{k}\right)}\right)\right|^{2}\left|N T F^{k}\left(e^{2 j \pi N_{d} f}\right)\right|^{2}$ for $k=4$. Most of the noise energy remaining after filtering lies between the modulator boundaries (vertical lines).

Thus it can be supposed that each FIR filter suppresses all the out-of-band noise, which leads to:

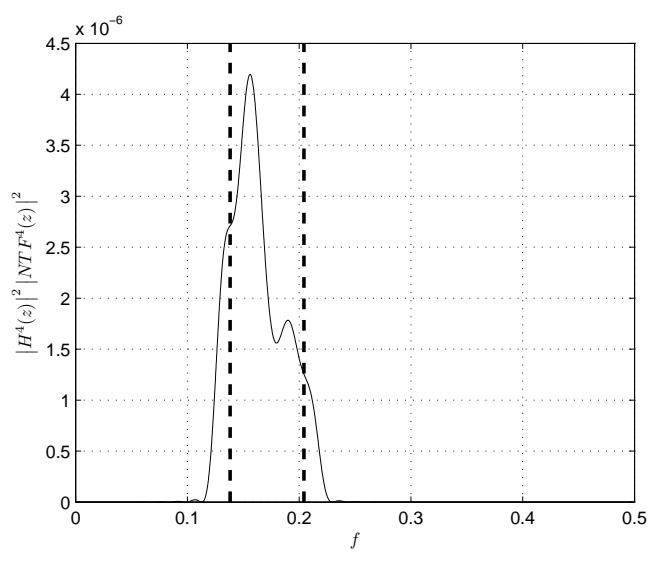

Fig. 3. Noise power spectrum density after filtering

$$
P_{N T F^{k}}=\int_{f=f_{r}^{k}}^{f_{r}^{k+1}}\left|N T F^{k}\left(e^{2 j \pi f}\right)\right|^{2} \Gamma(f) d f
$$

$f_{r}^{k}$ and $f_{r}^{k+1}$ being the actual boundaries for the use of modulator $k$,

$$
\Gamma_{k}(f)=\Gamma=\frac{1}{3 \times 4^{N b i t}},
$$

where Nbit is the number of bits of the ADC within each sigma delta modulator. All sub-bands are continuous, and $f_{r}^{0}=f_{1}, f_{r}^{N+2}=f_{2}$.

$f_{t}^{k}$ are constant frequencies chosen as the middle of the band of each modulator $k$; the global noise power can be expressed:

$$
P_{N T F_{t}}=\Gamma \sum_{k=0}^{N+1} \int_{f=f_{r}^{k}}^{f_{r}^{k+1}}\left|N T F^{k}\left(e^{2 j \pi f}\right)\right|^{2} d f
$$

Each integral is split into two terms that have at least one constant boundary. The first and the last term in the sum are separated as they are constant.

$$
\begin{aligned}
& P_{N T F_{t}}=\Gamma \int_{f=f_{1}}^{f_{t}^{0}}\left|N T F^{0}\left(e^{2 j \pi f}\right)\right|^{2} d f
\end{aligned}
$$

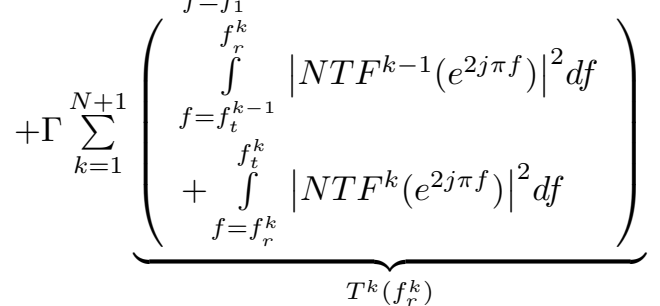

$$
\begin{aligned}
& +\Gamma \int_{f=f_{t}^{N+1}}^{f_{2}}\left|N T F^{N+1}\left(e^{2 j \pi f}\right)\right|^{2} d f
\end{aligned}
$$

The total noise power is then the sum of two constant values, plus $N+1$ terms, each of them depending on one 
of the boundaries $f_{r}^{1}$ to $f_{r}^{N+1}$. Each boundary can then be optimized independently from the others. Fig. 4 shows as an example $\left|N T F^{3}\right|,\left|N T F^{4}\right|$ and the term in Eq. (5) where $f_{r}^{4}$ is involved. The criterion appears reasonably convex and smooth.

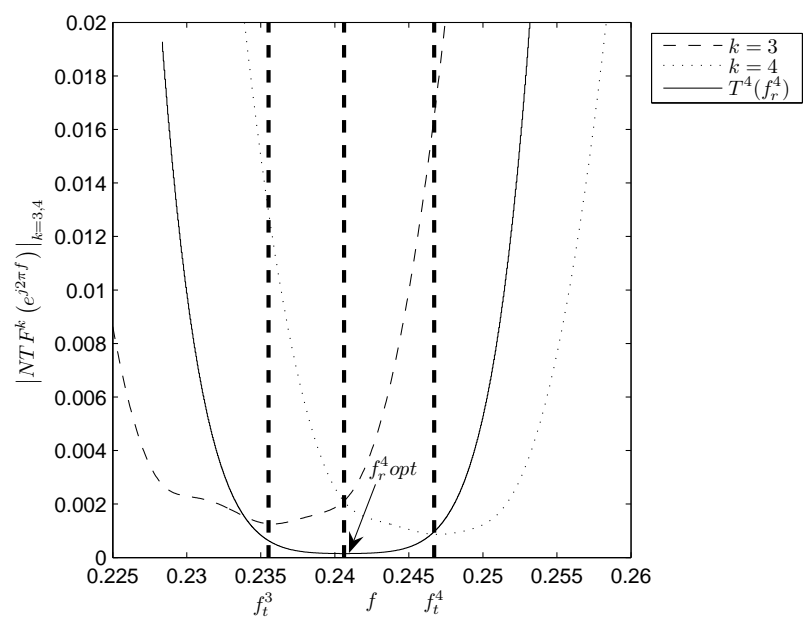

Fig. 4. Boundary optimization criterium

It was verified that this property remains true when taking into account the FIR filter effect, resulting in a behavior similar to that of Fig. 4.

\section{NOISE TRANSFER FUNCTION CALIBRATION ALGORITHM}

The noise transfer function is optimized by tuning the boundaries between modulators $f_{r}^{k}, k=1 . . N+1$ from $f_{r}^{k-1}$ to $f_{r}^{k+1}$. The frequencies used for the modulator and demodulator are deduced from these values:

$$
f_{C}^{k}=\left(f_{r}^{k-1}+f_{r}^{k}\right) / 2,
$$

and the bandwidths of the lowpass filters.

$$
\Delta f_{k}=\frac{f_{r}^{k}-f_{r}^{k-1}}{2} \text { for } k=1 . . N
$$

It was shown in [5] that an error of $4 \%$ in the width of the subband $(0.05 \%$ of the sampling frequency) causes a resolution loss less than 0.1 bit. Thus, the boundary frequency values $\left(f_{r}^{k}\right)$ can be quantized in this example with a step $q_{s}=F_{s} / 1024$. The whole band represents 102 steps and the bandwidth of each subband would be 12 or 13 steps if all modulators were ideal.

The input of the EFBD must be first grounded. The noise power produced by the quantization is minimized by varying the values of the boundary frequencies $f_{r}^{k}$. The demodulation sequence and the lowpass FIR filters are fully determined by the knowledge of these values ((6) and (7)).

The adaptation scheme given in Fig. 5 was used. The scheme itself uses the processing of the digital part presented in Fig. 1. The estimate of the converter noise power is:

$$
\hat{P}=\frac{1}{N_{s}} \sum_{n=1}^{N_{s}} \sum_{k=1}^{N+2}\left(I_{k}[n]^{2}+Q_{k}[n]^{2}\right)
$$

$N_{s}$ is the number of samples used for the power estimation, $I$ and $Q$ are the outputs of each filter (Fig. 1) .

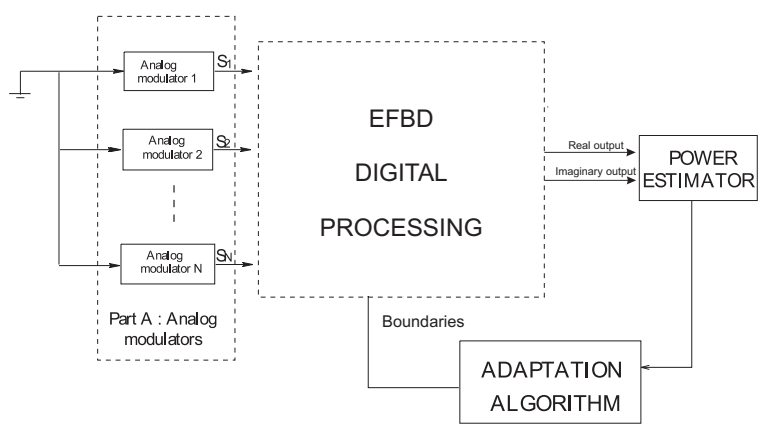

Fig. 5. Quantization noise minimization

The proposed algorithm is based on a so-called relaxation method. It does not require any operations excepted the calculation of the output power. Other faster algorithms could be used [9], but lead to more complex logic. Initial values are those obtained theoretically (i.e. design typical values). The algorithm changes iteratively the boundary value $f_{r}^{k}$ between $f_{r}^{k-1}$ and $f_{r}^{k+1}$ for $k=1$ to $N+1$ in each sequence. The first boundary remains $f_{1}$ and the last boundary remains $f_{2}$. This algorithm can be expressed for one sequence as:

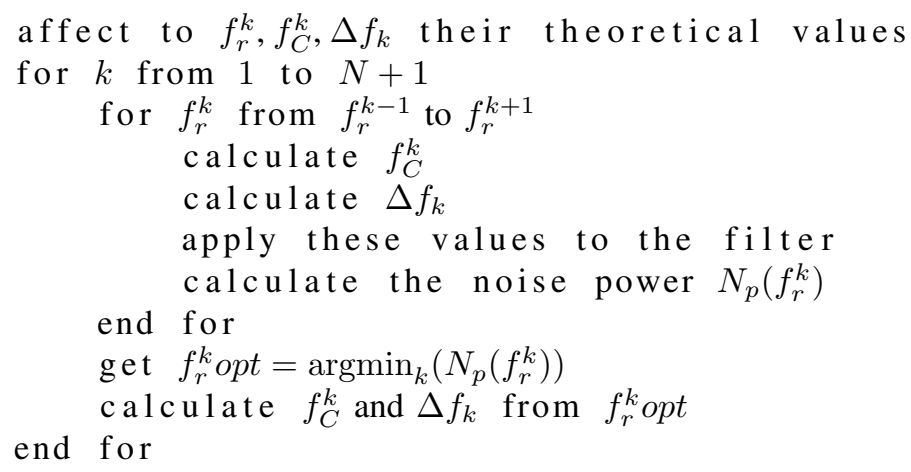

The output power is estimated for each boundary value and the value that minimizes the output power is memorized.

The quantization noise power is estimated from a finite number of samples, leading to an error in the estimation. Due to this error, the algorithm will not converge to the optimum value in one step. It has to be applied several times to be sure to reach the optimum point. When $2^{12}$ samples are used for the quantization noise power calculation, two or three sequences are required to reach the optimum.

This algorithm has been performed on a simulation of the bank described in Fig. 2 with 10 modulators. The output signals of the modulators have been memorized so that the same outputs are used for each loop. The results are given on Fig. 6, which shows the resolution of the EFBD (calculated from the estimated noise power) after each iteration. It may 
be seen that the optimum value is reached at the end of the first sequence.

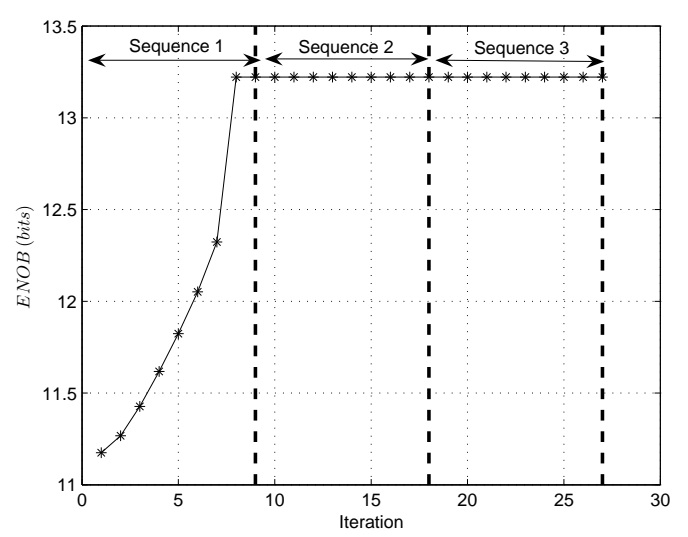

Fig. 6. Evolution of noise power during iterations -off line-

This algorithm has also been tested in a simulation where the outputs of the modulators were calculated for each iteration. The result is given in Fig. 7. It can be seen that the optimum is reached after three sequences. We verified by simulations that the convergence to the optimum value is also reached after three sequences for all kind of mismatches (with systematic or random errors on the modulator's central frequencies). The method is, therefore relevant.

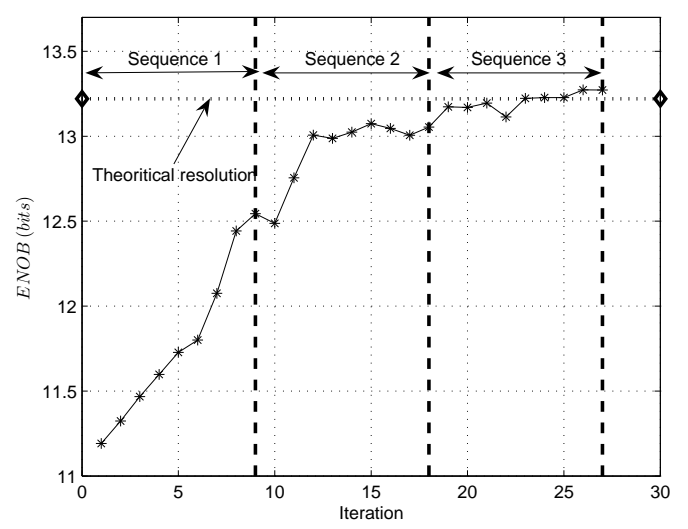

Fig. 7. Evolution of noise power during iterations -on line-

With an $800 \mathrm{MHz}$ analog frequency, each noise power calculation performed from 4000 samples takes $5 \mu$ s to perform (the OSR of the system is equal to 5), and each sequence in the worst case approximately $1.12 \mathrm{~ms}$ ( 9 boundaries are varied among 25 values). The total NTF calibration time for 3 sequences can be estimated to $3.3 \mathrm{~ms}$.

\section{CONCLUSION}

This paper describes an auto-calibration algorithm for an EFBD sigma-delta converter. The main advantage of this algorithm is to use the implemented digital filter plus a reasonable amount of logic. A power estimator, associated with counters and a sequencer is needed. The logic quantity associated with this estimator can be neglected compared with the main filter. The whole calibration process proposed in this paper can be performed in few ms, which is compatible with the power-up time of any communication system. Anyway, the possibility of performing a power-up adjustment have shown the feasibility of a EFBD converter, without requiring a strict analog trimming.

\section{REFERENCES}

[1] P. Aziz, H. Sorensen, and J. Van der Spiegel, "Multiband sigma-delta modulation," Electronics Letters, pp. 760-762, April 1993.

[2] P. Aziz, H. Sorensen, and J. Van der Spiegel, "Multiband sigma-delta analog to digital conversion," ICASSP, vol. 3, pp. 249-252, April 1994.

[3] A. Eshraghi and T. Fiez, "A comparative analysis of parallel delta-sigma ADC architectures," IEEE Trans. Circuit and Sys.I, vol. 51, pp. 450-458, March 2004.

[4] R. Schreier and G.C. Temes, Understanding Delta-Sigma Data Converters. New Jersey: Wiley, 2005, chap. 4.

[5] P. Benabes, A. Beydoun, and R. Kielbasa, "Dimensionnement prliminaire d'un banc de convertisseurs sigma-delta parallles," Taisa 2005, pp. xxx, 20052007.

[6] V. Ferragina, A. Fornasari, U. Gatti, P. Malcovati, F. Maloberti, "Gain and offset mismatch calibration in time-interleaved multipath A/D sigma-delta modulators," IEEE Transactions on Circuits and Systems I: Fundamental Theory and Applications, vol. 51, pp. 2365-2373, Dec. 2004.

[7] A. Delic-Ibukic, D.M. Hummels, "Continuous gain calibration of parallel delta sigma A/D converters," IEEE Instrumentation and Measurement Technology Conference, pp. 905-909, April 2006.

[8] R.D. Batten, A.Eshraghi, T.S. Fiez, "Calibration of parallel $\Sigma \Delta$ ADCs," IEEE Transactions on Circuits and Systems II: Analog and Digital Signal Processing, ,vol. 49, pp. 390-399, June 2002.

[9] W.H. Press, B.P. Flannery, S.A.Teukolsky, W.T.Vetterling, NUMERICAL RECIPES, The art of scientific computing. Cambridge University Press 1986.

[10] W.R. Bennett, "Spectra of quantized signals," Bell syst. tech. J., vol. 27, pp. 446-472, 1948.

[11] G. Appel, "Fractional N synthesizers," R.F. Design, vol. 23, pp. 34-50, Nov. 2000.

[12] P.V. Brennan,R. Walkington, "Stored-sequence sigma-delta fractional-N synthesizer," IEE Circuits, Devices and Systems, vol. 151, n 2, pp. 69-73, April 2004.

[13] M. Ortmanns and F. Gerfers, Continuous-Time Sigma-Delta A/D Conversion. Berlin: Springer, 2006.

[14] C. Lelandais-Perault, P. Benabes, J.L. De Gouy, and R. Kielbasa, "A parallel structure of a continuous-time filter for band-pass sigma-delta A/D converters," 10th IEEE International Conference on Electronics, Circuits and Systems, pp. 14-17, December 2003.

[15] S. Benabid, E. Najafi-Aghdam, P. Benabes, S. Guessab, R. Kielbasa, "CMOS design of a multibit bandpass continuous-time sigma delta modulator running at $1.2 \mathrm{GHz}$," IEEE International Caracas Conference on Devices, Circuits and Systems, pp. 51-55, November 2004. 